\title{
DEL POSMODERNISMO AL POSHUMANISMO: PRESENTE Y FUTURO DEL CONCEPTO DE HIBRIDEZ EN LA LITERATURA LATINOAMERICANA
}

From postmodernism to posthumanism: Present and future of the concept of hybridty in Latin American literature

\section{NICOLÁS BALUTET \\ Université Polytechnique Hauts-de-France (Francia) nicolas.balutet@uphf.fr}

Para calificar la estética posmodernista, que responde a la entrada en la era posmoderna, marcada por la ausencia de una visión unitaria y global del mundo, los teóricos de los años 70 privilegiaron el concepto de "heterogeneidad", que en la década siguiente y parte de los años 90, dejó paso al de "sincretismo", "mestizaje" o "creolidad" (en las Antillas) (Amselle, 1990; Gruzinski, 1999). A partir de los años 90 y, sobre todo, desde la primera década del tercer milenio, el concepto de "hibridez" ocupa un lugar destacado debido, en parte, a la influencia de los estudios poscoloniales (Ashcroft, Griffiths y Tiffin, 1989, p. 118; Toro, 2009, p. 27). Uno de los primeros en utilizar dicho concepto, en 1990, fue el crítico cultural argentino Néstor García Canclini (2010), pero no profundizó en la hibridez de las obras literarias. Por consiguiente, es preciso definir, en primer lugar, esta noción proteiforme, utilizada en nuestros días en numerosos campos (nuevas tecnologías, biología, genética, estrategia, política, economía, management, informática, artes, etc.) (Herd, 2009, p. 7; Sécardin, 2008, p. 23; Budor y Geerts, 2004, pp. 8-9; Molinet, 2006; Gambier, 2011, p. 7).

El término "hibridez" procede del latín ibrida, 'sangres mezcladas', alterado en hybrida por su similitud con el griego hybris, 'exceso, violencia, orgullo, desmesura' (Ezquerro, 2005, p. 9; Godin, 1996, p. 37; Ramos-Izquierdo, 2005, p. 61). Es una palabra tomada de los campos de la biología y la botánica, donde designa el "cruce de variedades, razas, especies diferentes"1 (Petit Robert 2010, 2009). El maíz, la nectarina, el mulo o el burdégano son algunos ejemplos de híbridos. Por extensión, el término significa comúnmente lo que "se compone de dos elementos de naturaleza diferente anormalmente reunidos; que participa de dos o varios conjuntos, géneros, estilos"2 (Petit Robert 2010, 2009). Este origen biológico y zoológico de la palabra explica que algunos investigadores

\footnotetext{
${ }^{1}$ El Trésor de la Langue Française Informatisé (2011) propone una definición similar: "cruce natural o artificial de dos individuos (plantas o animales) de especies, razas o variedades diferentes"; el Diccionario de la Real Academia Española (2011) también: "1. adj. Dicho de un animal o de un vegetal: Procreado por dos individuos de distinta especie; 2. adj. Biol. Dicho de un individuo: Cuyos padres son genéticamente distintos con respecto a un mismo carácter; 3 . adj. Se dice de todo lo que es producto de elementos de distinta naturaleza".

${ }^{2}$ Para el Grand dictionnaire Larousse (2008), el híbrido, en sentido figurado, es lo que está "compuesto de elementos discordantes".
} 


\section{Nicolás Balutet}

se hayan negado en ocasiones a utilizar dicho concepto, que en los siglos XVIII y XIX fue empleado por teorías racistas que presuponían la existencia de un estado original y puro (Louviot, 2005, p. 488; Young, 1995, pp. 6-9; Hall, 2009, p. 29):

Fue en gran parte a causa del racismo científico del siglo XIX por lo que la hibridación se codificó como una categoría negativa. En aquel momento, la ciencia creó la idea de "raza pura", una forma extremadamente falaz y aberrante de clasificación humana, nacida de la obsesión monoteísta occidental por el "Uno" y el "Mismo". Como resultado de los encuentros y enfrentamientos coloniales, la espinosa cuestión del mestizaje comenzó a alimentar la imaginación de los europeos con los fantasmas de la monstruosidad y la degeneración. [...] Identificando raza con especie, los poligenistas infirieron que los cruces entre diferentes razas [...] serían estériles o producirían híbridos estériles [...]. Evidentemente, la experiencia demostró incluso entonces que las "razas" humanas no constituían "especies", lo que podía encajar en ese modelo científico de la "hibridación". Pero la aversión de la sociedad decimonónica hacia la mezcla interracial o las "uniones no naturales" llevó a muchos científicos a conceptualizar "hibridación" como monstruosidad, decadencia y deterioro. (Lionnet, 1991, pp. 9-10).

Sin embargo, parte de la crítica actual prefiere utilizar el concepto de "hibridez" en lugar del de "mestizaje" - del latín mixus, 'mezclado'-, muy de moda en los 90, porque lo considera menos relacionado con la idea de "raza" y la historia colonial (Kuortti y Nyma, 2007; Lüsebrink, 1992-1993; Montandon, 2005). Además, al valorizar extremadamente al ser mestizo, como hacen los trabajos pertinentes al mestizaje, se puede dar la impresión de que la existencia mestiza constituye la más alta forma de existencia humana... una forma de racismo al revés ${ }^{3}$. Más que otras nociones, la hibridez se ve ahora como un "complejo" integrador, un hiperónimo capaz de innovar en cuanto a significación y forma y que es portador de tolerancia (Sécardin, 2008, pp. 22-23).

La estética posmodernista se caracteriza por la deconstrucción del canon único y las estructuras tradicionales de poder, la heterogeneidad de los géneros y discursos y las nuevas visiones del tiempo, del espacio y de los personajes (Balutet, 2013). El concepto de hibridez nos parece, por tanto, la herramienta idónea para explicar ciertos fenómenos de la escritura posmodernista. Lo más evidente y quizás lo más analizado por la crítica, la hibridez genérica, consiste en el estallido de los géneros. Podemos analizar también la hibridez dialógica, es decir, la transformación en un texto determinado de diferentes elementos culturales, literarios y linguísticos tomados de otros textos (intertextualidad, cita, alusión literaria, plagio, recuperación de un mito, etc.). Entran en esta categoría todos los fenómenos de reescritura, es decir, la imitación (parodia, pastiche) o la translación

\footnotetext{
${ }^{3}$ En cuanto al concepto de "sincretismo", se reserva casi siempre para designar los fenómenos de fusión de movimientos religiosos o simbólicos (García Canclini, 2009, pp. 78-79).

324 | Alpha No50 (Julio 2020) PÁGS. 323-334. ISSN 07 16-4254
} 
(traducción, transcripción, adaptación), así como la polifonía y el plurilingüismo. En efecto, en un texto literario, el lenguaje pocas veces resulta uniforme y puede, por el contrario, integrar varios registros, incorporar rasgos de otras lenguas mediante préstamos léxicos o sintácticos, diálogos, citas de autores extranjeros. Hemos decidido hablar de "hibridez dialógica" porque todos los fenómenos evocados aquí, cualquiera que sea el nombre que se les dé, fueron estudiados primero por Mijaíl Bajtín que desarrolló la noción de dialogismo. Por otra parte, dicho concepto refleja perfectamente la idea de que se entablan diálogos con otros textos, con otros idiomas, con otras artes. Asimismo, Mijaíl Bajtín, en Estética y teoría de la novela, utiliza varias veces la noción de "hibridación" y el término "híbrido" (1978, pp. 125-126, 175-176, 182)4. Es interesante notar también que Antoine Compagnon, al referirse a la arquitectura posmodernista, la califica de "dialógica" (1990, p. 151) porque juega con formas y elementos heterogéneos, procedentes de diversas tradiciones.

El tercer nivel de hibridez corresponde a las nociones básicas (tiempo, espacio, personajes). El tiempo resulta híbrido mediante la coexistencia y la fusión de varias esferas temporales (anacronismos, sueños, rupturas diversas), y el espacio se caracteriza por una superposición de lugares antinómicos o simplemente distintos, pero ambos reflejan el sentir de los personajes. Si la hibridez constituye "la fuerza creadora de la reunión" (Budor y Geerts, 2004, p. 13) por la coexistencia de elementos discordantes, puede conllevar asimismo dificultades para conciliarlos armoniosamente. Los personajes aparecen, entonces, como seres contradictorios, paradójicos, desgarrados, inestables (Louviot, 2010, p. 228). Los textos posmodernistas privilegian ciertas figuras en particular. Debido a que la pérdida de los antiguos valores, característica de la era posmoderna, afecta a la familia, surgen hijos ilegítimos, personajes amenazados por el entorno familiar y huérfanos (pp. 72-73). Solemos encontrar también figuras de exiliados que exploran la distancia con su comunidad de origen a la que ya no pertenecen completamente (pp. 503-506), y todos aquellos que transgreden las fronteras de clase, etnia o género, así como las que separan lo humano y lo animal, lo muerto y lo vivo. De hecho, la hibridez nos parece una noción notoriamente relacionada con la identidad que hoy se define respecto del género, la etnia y la sexualidad (Larraín, 1996, p. 50).

Los estudios poscoloniales utilizan mucho el concepto de hibridez para denunciar el discurso colonial, que concibe el mundo como dos polos antagónicos y fijos: por un lado, el centro, correspondiente a la metrópolis situada en Occidente, que se autocalifica de civilizado; por otro lado, la periferia, en el "Tercer Mundo", definida como primitiva y salvaje. En este contexto, se excluía al sujeto colonizado, visto como el "otro", se concebían las identidades y las culturas como puras, es decir, sin ninguna mezcla, y se

${ }^{4}$ El término "hibridación" utilizado por Mijaíl Bajtín nos parece más restringido que el de "hibridez" por focalizarse sobre todo en la idea de proceso y menos en la de resultado. 


\section{Nicolás Balutet}

pugnaba por mantener las fronteras que separaban los dos polos antitéticos (Hardt, 1995, p. 21; Bhabha, 2007, p. 121). Los estudios poscoloniales proponen, al contrario, una visión del mundo colonial, ya no dividido en estructuras binarias que se excluyen mutuamente, sino marcado por movimientos incesantes de interpenetración, por la ambivalencia antes que por la simple y constante oposición (Rémy, 2011). Homi Bhabha los llama "espacios", "intersticiales" (2007, p. 30) o "tercer espacio"5 (p. 80). Uno $u$ otro se sustituyen, al mismo tiempo, por uno y otro y $n i$ uno $n i$ otro:

si la hibridez es importante, no lo es porque permitiría recobrar dos momentos originales a partir de los cuales emergería un tercero; para mí, la hibridez es más bien el 'tercer espacio' que hace posible la emergencia de otras posiciones. Este tercer espacio viene a perturbar las historias que lo constituyen y establece nuevas estructuras de autoridad, nuevas iniciativas políticas, que escapan al sentido común (Bhabha, 2006, p. 99).

La identidad que surge de esta situación se caracteriza, pues, por una "hibridez cultural" (Bhabha, 2007, p. 33), una hibridez hecha de construcciones, negociaciones, reapropiaciones que se sitúa en un "más allá" de las categorías binarias, en una superación de las oposiciones (Toro, 2006, p. 224). Al proponer un "más allá", es decir, una alternativa a los conceptos tradicionales de singularidad y totalidad, la noción de hibridez se focaliza en la diferencia y la diversidad, reconociendo la multiplicidad identitaria del sujeto exiliado, inmigrante o desplazado, y rechazando el esencialismo, lo que permite extender el polo de la periferia a todos los márgenes: los marginados en función de su etnia, pero también de su género y de su preferencia sexual (Bhabha, 2007, p. 56; Deremetz, 1996, pp. 5-6; Le Bourdellès, 1996; Sherry, 1999, p. 32; Toro, 2006, p. 223).

Como escribíamos al principio de este artículo, la noción de hibridez ha generado mucho debate en las últimas dos décadas y actualmente se emplea a veces de manera excesiva, para remitir a una multiplicidad de objetos dispares. Si este término resulta tan utilizado es porque parece designar eficazmente la era presente. En efecto, si nos quedamos en el ámbito literario, nos damos cuenta de que hace unos años las prácticas híbridas trastornaban nuestras costumbres de lectura y percepción mientras que hoy encajan naturalmente en nuestro sistema cognitivo y epistemológico (Paterson, 2001, p. 91). La escritura híbrida podría representar, por tanto, la escritura de nuestro tiempo. Este mundo se ha visto radicalmente modificado desde la segunda mitad del siglo XX por el fenómeno de la globalización, que constituye un nuevo modo de relación entre las culturas y permite una mezcla de elementos procedentes de diferentes sociedades. Por mucho que dichos intercambios no se operen siempre de manera pacífica y descansen en procesos de negociación y compromiso, no cabe duda de que se generan síntesis

\footnotetext{
${ }^{5}$ Un excelente resumen de las teorías desarrolladas por Homi Bhabha se encuentra en Saki (2011, pp. 233-240). 326 | Alpha No50 (Julio 2020) PÁGS. 323-334. ISSN 07 16-4254
} 
interétnicas en todos los ámbitos, desde lo humano -mediante el mestizaje- hasta los productos culturales, más aún debido al desarrollo de los medios de transporte y de Internet en los últimos años (Smith, 2006, p. 376). Algunos afirman incluso que gracias a los progresos de la ciencia el hombre se está convirtiendo en un ser humano "aumentado", en un nuevo individuo híbrido, mitad hombre, mitad máquina, por la presencia de elementos electrónicos, ya no sólo en la ropa, sino introducidos en el cuerpo mediante chips, un hombre genéticamente modificado, casi perfecto, gracias a un cuerpo sin edad, un cerebro infalible, una reproducción controlada... En definitiva, un homo tecnologicus o cyborg, que vemos encarnado ya en el atleta sudafricano, estrella de los últimos Juegos Olímpicos de Londres, Oscar Pistorius. Resulta sumamente interesante esta figura del cyborg en la medida en que parece simbolizar, tanto en su aspecto material como en el imaginario, un paso adelante en el concepto de hibridez desarrollado por la estética posmodernista.

El cyborg ("cybernetic organism"), término creado en 1960 por dos investigadores de la NASA, Manfred Clynes y Nathan Kline, designa la fusión de un organismo vivo -en general un ser humano-y determinadas tecnologías con el fin de mejorar o sustituir algunas funciones orgánicas. Esta figura, recuperada y popularizada por el cine (Blade Runner, Terminator, Robocop, Inteligencia artificial, Yo Robot, por citar las películas más famosas) y la literatura de ciencia-ficción (El hombre bicentenario, de Isaac Asimov; Neuromante, de William Gibson; Flores para un cyborg y Las criaturas del cyborg, de Diego Muñoz Valenzuela; Ygdrasil, de Jorge Baradit; etc. $)^{6}$, está cobrando cada vez más importancia en la vida actual, porque lo que hace pocos años parecía mera fantasía hoy empieza a hacerse realidad. En efecto, actualmente no solo nuestra vida es inconcebible sin la tecnología, sino que los objetos que nos rodean van formando parte cada vez más de nuestro propio cuerpo. Para algunos pensadores, el homo cyborg no es una novedad en la medida en que, desde el comienzo de la humanidad, el hombre ha transformado "su medio con herramientas y objetos artificiales" y ha establecido "relaciones de control y comunicación entre el cuerpo y [este] medio" (Yehya, 2010, p. 150). Desde este punto de vista, unas gafas, un marcapasos, una prótesis de cadera y hasta la ropa ya nos hibridizarían. Sin llegar a estos extremos, es importante reconocer que estamos experimentando una verdadera transición con el desarrollo de la cirugía plástica, la robótica, la biotecnología, la nanotecnología, etc., asociado a los avances de las nuevas tecnologías de la información y la comunicación. Esta

\footnotetext{
${ }^{6}$ Dentro de la ciencia-ficción, las novelas que utilizan la figura del cyborg y, en general, la alta tecnología, pertenecen a la corriente denominada "ciberpunk" (Grugnier, 2003, p. 227; Muñoz Zapata, 2009). Podemos ver en Edgar Allan Poe un precursor de esta corriente ya que, en su cuento The man that was used up (1839), describe a un hombre dotado de prótesis mecánicas. En el ámbito latinoamericano, Andrew Brown (2010, p.1) evoca Horacio Kalibang o los autómatas (1879), del argentino Eduardo Holmberg, Hombre artificial (1909), del uruguayo Horacio Quiroga, así como Hombre y engranajes (1951) y Rayuela (1963) de los argentinos Ernesto Sábato y Julio Cortázar, respectivamente.
} 
"robolución" se está produciendo, a pesar de todo, bastante gradualmente, lo que transmite la impresión de que la figura del cyborg es y seguirá siendo una quimera. Sin embargo, de acuerdo con Jeffrey Deitch, consideramos que "muchas de las nuevas actitudes hacia el cuerpo y los nuevos modos de comportamiento social no parecen particularmente significativos de forma aislada, pero vistos en conjunto muestran una marcada tendencia hacia un modelo radicalmente nuevo de comportamiento individual y social ..." (Burrows, 1995). Dicho modelo no es más que la poshumanidad ${ }^{7}$.

Este término, algo inquietante, remite a la imagen utópica de un futuro, quizás no tan lejano, en el que se habrá operado una revisión del concepto tradicional de lo humano. En ese mundo teorizado por Donna Haraway en su Manifiesto para cyborgs [1985], las fronteras entre el organismo vivo y la tecnología han desaparecido para dejar paso al poshumano, un ser híbrido que se aleja de la típica y común figura varonil del cyborg presente en las novelas, en los medios de comunicación y, sobre todo, en las películas estadounidenses (Grugier, 2003, p. 225). La fusión en un mismo cuerpo de lo orgánico y lo mecánico ${ }^{8}$ ha hecho trascender numerosas dualidades que "contribuyen a la lógica y a las prácticas del sistema de dominación de las mujeres, de la gente de color, de la naturaleza, de los trabajadores y de los animales; en pocas palabras, la dominación de todo lo que constituye al otro" (Haraway, 2007, p. 75). En este mundo, la idea misma de "naturaleza", por ejemplo, ha desaparecido por representar un espejismo reaccionario, una vuelta a un paraíso perdido. Lo mismo ocurre con el género, ya que las fronteras entre máquina, animal (con los trasplantes de órganos) y ser humano han sido desdibujadas. En cuanto a la orientación sexual, tampoco tiene sentido porque el cyborg no se reproduce sino que se replica: "el sexo del cyborg restaura algo de la hermosa libertad replicativa de los helechos y de los invertebrados [...]. La replicación del cyborg no precisa reproducción orgánica" (Haraway, 2007, p. 30). Los avances actuales en cuanto a procreación sexuada como la fecundación in vitro, las madres portadoras, el útero artificial o la clonación (Robin, 2000b, pp. 188-189) podrían hacer realidad en un futuro más o menos próximo lo que anuncian tanto Donna Haraway como Jean-Louis Touraine, profesor de medicina: "Pasado mañana, estoy seguro de ello, el desarrollo fetal podrá desarrollarse in vitro, en una especie de incubadora especial, desde su origen hasta su final. [...] El nacimiento existirá sin embarazo. Y no cabe duda de que, cuando este método se haya desarrollado, tenderá a generalizarse. [...] Nada podrá oponerse eficazmente a esta progresión" (1985, pp. 226-228).

\footnotetext{
${ }^{7}$ Algunas vías hacia la poshumanidad pueden leerse en Robitaille (2007, pp. 17-95).

${ }^{8}$ Katherine Hayles explica que, "en el poshumano, no existen diferencias esenciales o demarcaciones absolutas entre la existencia física y la simulación por ordenador, el mecanismo cibernético y el organismo biológico, la teleología robot y los objetivos humanos" (1999, p. 3).

328 | Alpha No50 (Julio 2020) PÁGs. 323-334. ISSN 07 16-4254
} 
En resumidas cuentas, la poshumanidad, al borrar toda una serie de dicotomías, autorizaría el verdadero advenimiento de la multiplicidad, la indeterminación, la inmaterialidad. Aunque estos cambios puedan parecer positivos por ofrecer una liberación total de la corporeidad, este extreme make-over ${ }^{9}$ del ser humano deja en el aire la interrogación acerca de lo que nos hace humanos y refuerza el temor a la superación del hombre por la máquina en un proceso desigual de mecanización del hombre y de humanización de las máquinas. La tradicional figura literaria del monstruo, que aborda las relaciones entre diversos seres y especies y que pone en duda las fronteras del ser humano, ya refleja nítidamente esta inquietud.

Tanto estas interrogaciones como sus manifestaciones utópicas recordadas por Donna Haraway han dejado de ser confinadas en el campo restringido de la literatura de ciencia-ficción. En efecto, en los últimos años asistimos al auge de obras de ficción basadas en la nueva realidad de las tecnologías ${ }^{10}$. Estas impregnan incluso las novelas de algunos escritores latinoamericanos que hasta ahora no habían manifestado un interés particular por ellas. Quizás les parezca indispensable referirse al cyborg porque se ha convertido en una figura epistemológica esencial para pensar la época contemporánea. Esa es la opinión de Philippe Quéau: "el ciberespacio no es solo una nueva Utopía, una nueva América abierta a los colonizadores y dispuesta a estructurarse según la voluntad de los empresarios de redes, también es el lugar de despliegue de una nueva manera de estar en el mundo, de pensar el mundo y de actuar sobre él" (1995, p. 66). Entre estos escritores, podemos citar a los argentinos Ricardo Piglia, Alicia Borinsky y Rodrigo Fresán, a los chilenos Eugenia Prado y Alberto Fuguet, al uruguayo Rafael Courtoisie, al boliviano Edmundo Paz-Soldán y a la mexicana Carmen Boullosa. Magníficamente estudiados en la obra pionera de Andrew Brown, Cyborgs in Latin America (2010) ${ }^{11}$, todos estos autores están diseñando una poética cibernética o poshumanista de la que queremos señalar algunos rasgos.

$\mathrm{Al}$ igual que el posmodernismo y otras estéticas anteriores, si el poshumanismo hace estallar los géneros literarios, que se vuelven aún más indefinidos, lo más llamativo, sin embargo, radica en la atención que se dedica al lenguaje, lo que no debe sorprender si constatamos, con David Porush, que los autores "se centran en la maquinaria o tecnología de su ficción, únicamente conscientes de que sus textos se construyen con palabras, de que las palabras forman parte de una maquinaria mayor que es el lenguaje, y

\footnotetext{
${ }^{9}$ Referencia a un famoso programa televisivo de cirugía plástica.

${ }^{10}$ Nos limitamos a evocar las obras impresas y no las obras en soporte digital, tan bien estudiadas por Alexandra Saemmer (2007) en un libro apasionante.

${ }^{11}$ El crítico analiza La ciudad ausente (1992), de Ricardo Piglia; Cine continuado (1997), de Alicia Borinsky; Mantra (2001), de Rodrigo Fresán; Lóbulo (1998), de Eugenia Prado; Por favor, rebobinar (1996), de Alberto Fuguet; Tajos (1998), de Rafael Courtoisie; Sueños digitales (2000) y El delirio de Turing (2003), de Edmundo Paz-Soldán; y Cielos de la tierra (1997), de Carmen Boullosa.
} 


\section{Nicolás Balutet}

de que el lenguaje está constituido por la maquinaria aún mayor de su propia conciencia y experiencia" (1985, p. 19). Este lenguaje se caracteriza por su extrema heterogeneidad. En efecto, al intentar transcribir las nuevas posibilidades ofrecidas por la tecnología como, por ejemplo, los foros de discusión de las comunidades virtuales, es decir, una forma de escritura sometida a la presión de la oralidad, o el hipertexto, esos documentos no jerarquizados relacionados entre sí por enlaces -una forma muy rizomática, para retomar un adjetivo de Gilles Deleuze y Félix Guattari ${ }^{12}$-, el lenguaje se hace complejo, múltiple, confuso, inestable, fragmentario... (Robin, 2000a, pp. 13-18; Taylor y Pitman, 2007). Como ejemplo de hipertextualidad, podemos recordar que el filósofo Thierry Hoquet hace uso de ella en la segunda parte de su interesante libro Cyborg Philosophie:

Los párrafos de esta parte se han agrupado en doce secciones que conforman otras tantas unidades temáticas. Podemos hacer una lectura lineal siguiendo el orden de la numeración. Cada párrafo sugiere además una bifurcación (Bif) hacia un párrafo de otra sección. Esta hipertextualidad artesanal permite perspectivas inéditas, a veces juegos de ping-pong, a veces saques de esquina, a veces pases distendidos, pero siempre nuevas resonancias entre textos desunidos. Por fin, también es posible una lectura aleatoria: este libro, como Cyborg, empieza por donde se quiera (Hoquet, 2011, p. 141).

Mediante este lenguaje, resuenan las palabras de Donna Haraway, para quien "la política cyborg lucha a favor del lenguaje, lucha contra la comunicación perfecta, contra ese código único que traduce a la perfección cada significado, el dogma central del falogocentrismo" (2007, p. 73). Por otra parte, el desarrollo de las redes y de lo virtual, ya señalado por los pensadores de la posmodernidad, con sus consecuencias en cuanto a la abolición de las distancias, la instantaneidad, la ubicuidad, la incorporeidad, la atemporalidad, etc., se ve ampliado en estas obras sobre el cyborg definido como un "entrelazado de membranas, que define a la vez superficies $y$ profundidades, toda una topología de intersecciones donde se inventan tantos interiores como exteriores, que engendran otras tantas polaridades, que suscitan otros tantos encuentros" (Hoquet, 2011, p. 227).

Como muestra este rápido repaso de algunas manifestaciones de la poshumanidad y de sus implicaciones literarias, podemos afirmar que volvemos a encontrar los ejes de la hibridez posmodernista en sus aspectos formales. Sin embargo, por muy difícil que sea evaluar una corriente literaria reciente, nos parece que el poshumanismo se manifiesta en una sobrecodificación de algunas de las características posmodernistas tal y como la escritura posmodernista lo hace respecto del Modernism que, en Hispanoamérica, abarca

\footnotetext{
${ }^{12}$ En Mil mesetas, los dos filósofos desarrollan el concepto de rizoma, que "no está hecho de unidades, sino de dimensiones, o más bien de direcciones cambiantes. No tiene ni principio ni fin, pero siempre tiene un medio por el que crece y se desborda" (1980, p. 31).

330 | Alpha No50 (Julio 2020) PÁGS. 323-334. ISSN 07 16-4254
} 
no solo las obras del boom sino también las novelas vanguardistas, existencialistas, fantásticas o real maravillosas (Balutet, 2013). El ejemplo de los hipertextos podría ilustrarlo. No se trata de un fenómeno nuevo, aparecido a mediados de los años 90 con el desarrollo de Internet, sino que los hipertextos ya existían en la literatura con anterioridad, desde las vanguardias y, sobre todo, desde el posmodernismo. Prueba de ello son los hipertextos creados por el OuLiPo (Ouvroir de Littérature Potentielle, "Taller de literatura potencial"), ese grupo internacional fundado en 1960 por el matemático François Le Lionnais y el escritor y poeta Raymond Queneau ${ }^{13}$.

Asimismo, es imprescindible señalar que la estética poshumanista difiere de la posmodernista en cuanto a la hibridez identitaria. Al contrario de lo que ocurre en el corpus posmodernista, parece que en el poshumanismo la identidad ya no se establece en función del género, de la orientación sexual y de la etnia. Se ha llegado a considerar a los seres humanos como un bloque homogéneo en el que las diferencias interhumanas ya no parecen plantear ningún problema o cuestionamiento. No queremos decir con ello que el poshumanismo deje de interrogarse acerca de la identidad. Se trata más bien de todo lo contrario, pero esa interrogación se ha desplazado hacia los nuevos acoplamientos entre máquina, hombre y animal. ¿Representa el cyborg a la humanidad perdida, entendida como la referencia de lo puro, lo libre y lo natural, o la esperanza de "un mundo purificado, sin eructos ni pedos, sin sudor ni mocos, sin mierda ni pis"? (Hoquet, 2011, p. 28). ¿Simboliza el cyborg "un mundo del que la enfermedad habrá sido desterrada, del que el envejecimiento se habrá proscrito, un mundo en el que las caras no se arrugarán, en el que las pieles no se plegarán, donde los alientos no apestarán, donde las barrigas no se ablandarán, donde los órganos no resonarán"? (pp. 28-29).

$\mathrm{Si}$ actualmente el concepto de hibridez sigue siendo operativo para analizar las obras pertenecientes al poshumanismo, una nueva etapa en la ya larga historia de la literatura, quizás pueda parecer también algo estrecho para explicar todos estos nuevos fenómenos poshumanistas. $\mathrm{Si}$, en principio, el cyborg sigue encarnando perfectamente al ser híbrido, hay que tener en cuenta que la hibridez en sí está superada, ya no aparece como algo raro u original. Además, el mundo ya no se sitúa en el "tercer espacio" de Homi Bhabha sino en infinidad de espacios-tiempo dentro de otros espacios-tiempo que multiplican las posibilidades ad infinitum. Cuando tengamos una mayor perspectiva acerca de las obras poshumanistas, probablemente surja la necesidad de encontrar nuevas herramientas conceptuales que permitan analizarlas mejor.

${ }^{13}$ Un estudio pormenorizado de los hipertextos del OuLiPo se encuentra en Saemmer (2007). AlPha № 50 (Julio 2020) PÁGs. 323-334. ISSN 07 16-4254| 331 


\section{OBRAS CITADAS}

Amselle, Jean-Loup (1990). Logiques métisses. Anthropologie de l'identité en Afrique et ailleurs. París: Payot.

Ashcroft, Bill, Gareth Griffiths y Helen Tiffin (1989). The Empire Writes Back. Theory and Practice in Post-Colonial Literatures. Nueva York: Routledge.

Bajtín, Mijaíl (1978). Esthétique et théorie du roman. París: Gallimard.

Balutet, Nicolas (2013). Poética de la hibridez en la literatura mexicana posmodernista (Laura Esquivel, Margo Glantz, Luis Zapata), Tesis de Doctorado, Colima, Centro de Estudios Superiores e Investigación.

Bhabha, Homi (2007). Les lieux de la culture: une théorie postcoloniale. París: Payot. (2006). "Le tiers-espace. Entretien avec Jonathan Rutherford", Multitudes, No 26 : 95-107.

Brown, Andrew (2010). Cyborgs in Latin America. Nueva York: Palgrave MacMillan.

Budor, Dominique y Walter Geerts (2004). Le texte hybride. París: Presses de la Sorbonne Nouvelle.

Burrows, R.J. (1995). “Cyberpunk as Social and Political Theory”, Cyberpunk Project

[http://project.cyberpunk.ru/idb/cyberpunk_as_socpolitical_theory.html]

(consultado el 7 de febrero de 2013).

Compagnon, Antoine (1990). Les cinq paradoxes de la modernité. París: Seuil.

De Toro, Alfonso (2009). Epistémologies. Le Maghreb. París: L'Harmattan.

(2006). 'Hacia una teoría de la cultura de la 'hibridez' como sistema científico 'transrelacional', 'transversal' y 'transmedial'", Cartografías y estrategias de la "postmodernidad" y la "postcolonialidad" en Latinoamérica, edición de Alfonso de Toro. Madrid: Iberoamericana, 195-242.

Deleuze, Gilles y Félix Guattari (1980). Mille plateaux. París: Les Éditions de Minuit.

Deremetz, Alain (1996). "Préface". Uranie, Nº: 5-6.

Diccionario de la Real Academia Española (2011). [http://lema.rae.es/drae].

Ezquerro, Milagros (2005). “Avant-propos", L'hybride/Lo hibrido. Cultures et littératures hispano-américaines, edición de Milagros Ezquerro. París: Indigo, 9-10.

Gambier, Yves (2011). "Introduction", Hybridité discursive et culturelle, edición de Eija Suomela-Salmi e Yves Gambrier. París: L'Harmattan, 7-23.

García Canclini, Néstor (2010). Cultures hybrides. Stratégies pour entrer et sortir de la modernité. Quebec: Presses de l'Université de Laval.

_ (2009). "Cultures hybrides et stratégies de communication”, Les identités collectives à l'heure de la mondialisation, edición de Bruno Ollivier. París: CNRS Éditions, 77-97.

Godin, Christian (1996). 'L'hybride entre la puissance et l'effroi', Uranie, № 6, 37-47.

Grugier, Maxence (2003). 'L'utopie cyborg. Réinvention de l'humain dans un futur surtechnologique", Revue Quasimodo, № 7, 223-238. 
Gruzinski, Serge (1999). La pensée métisse. París: Fayard.

Hall, Stuart (2009). "Une perspective européenne sur l'hybridation: éléments de réflexion", Les identités collectives à l'heure de la mondialisation, edición de Bruno Ollivier. París: CNRS Éditions, 29-36.

Haraway, Donna (2007). Le manifeste cyborg et autres essais, edición de Laurence Allard, Delphine Gardey y Nathalie Magnan. París: Exils Éditeur.

Hardt, Michael (1995). 'L'hybridité de l'Empire”, Futur Antérieur, Volumen 27, № 1, 19-33. Hayles, Katherine (1999). How We Became Posthuman: Virtual Bodies in Cybernetics, Literature and Informatics. Chicago: University of Chicago Press.

Hoquet, Thierry (2011). Cyborg Philosophie. Penser contre les dualisms. París: Seuil.

Kuortti, Joel y Jopi Nyman (2007). "Introduction: Hybridity Today", Reconstructing Hybridity. Post-Colonial Studies in Transition, edición de Joel Kuortti y Jopi Nyman. Ámsterdam: Rodopi, 1-18.

Larraín, Jorge (1996). "Posmodernismo e identidad latinoamericana", Escritos. Revista del Centro de Ciencias del Lenguaje, Núm. 13-14, 45-75.

Le Bourdellès, Hubert (1996). "L'hybridité culturelle dans les débuts du Moyen Âge occidental", Uranie, № 6, 103-112.

Louviot, Myriam (2010). Poétique de l'hybridité dans les littératures postcoloniales, Tesis de Doctorado. Estrasburgo: Universidad de Estrasburgo.

(2005). "L'hybridité, un concept pour aborder les littératures post-coloniales", Métissages littéraires, edición de Yves Clavaron y Bernard Dieterle, Saint-Etienne, Publications de l'Université de Saint-Etienne, 487-493.

Lüsebrink, Hans-Jürgen (1992-1993). "Métissage: Contours et enjeux d'un concept carrefour dans l'aire francophone", Etudes Littéraires, Volumen 25, № 3, 93-106.

Molinet, Emmanuel (2006). "L’hybridation: un processus décisif dans le champ des arts plastiques", Le Portique, $\mathrm{N}^{\circ} 2$.

Montandon, Alain (2005). "Préface", Métissages littéraires, edición de Yves Clavaron y Bernard Dieterle. Saint-Etienne: Publications de l'Université de Saint-Etienne, 7-10.

Muñoz Zapata, Juan Ignacio (2009). Le cyberpunk vernaculaire de l'Amérique latine: dystopies, virtualités et résistances, Tesis de Doctorado. Montreal: Universidad de Montreal.

Paterson, Janet (2001). 'Le paradoxe du postmodernisme. L'éclatement des genres et le ralliement du sens", Enjeux des genres dans les écritures contemporaines, edición de Robert Dion, Frances Fortier y Elizabeth Haghebaert. Quebec: Nota Bene, 81-101.

Petit Robert 2010 (2009). París: Le Robert.

Porush, David (1985). This Soft Machine: Cybernetic Fiction. Nueva York: Metheun.

Quéau, Philippe (1995). "Le virtuel, un état du réel”, Virtualité et réalité dans les sciences, edición de Gilles Cohen-Tannoudji. París: Frontières, 61-93.

Ramos-Izquierdo, Eduardo (2005). "De lo híbrido y de su presencia en la novela mexicana AlPHA No 50 (Julio 2020) PÁGs. 323-334. ISSN 07 16-4254| 333 
actual', L'hybride/Lo hibrido. Cultures et littératures hispano-américaines, edición de Milagros Ezquerro. París: Indigo, 59-104.

Rémy, Julien (2011). "Sur les postcolonial studies: hybridité, ambivalence et conflit", Revue du MAUSS permanente. Disponible en:

[http://www.journaldumauss.net/spip.php?article801]

Robin, Régine (2000a). "Le texte cyborg", Études françaises, Volumen 36, No 2, 11-38.

— (2000b), "Du corps cyborg au stade de l'écran”, Communications, № 70, 183-207.

Robitaille, Antoine (2007). Le Nouvel Homme nouveau. Voyage dans les utopies de la posthumanité. Quebec: Boréal.

Saemmer, Alexandra (2007). Matières textuelles sur support numérique. Saint-Etienne: Universidad de Saint-Etienne.

Saki, Mohamed (2011). "Hybridité, créolisation et la question de l'homogène et du divers", Hybridité discursive et culturelle, edición de Eija Suomela-Salmi e Yves Gambrier. París: L'Harmattan, 231-249.

Sécardin, Olivier (2008). Sémiocritique de l'hybridité. Du moderne au postmoderne, Tesis de Doctorado. París: Universidad París-Sorbona.

Sherry, Simon (1999). Hybridité culturelle. Montreal: L'île de la Tortue.

Smith, Andrew (2006). "Migrance, hybridité et études littéraires postcoloniales", Penser le postcolonial. Une introduction critique, edición de Neil Lazarus. París: Éditions Amsterdam, 359-386.

Taylor, Claire y Thea Pitman (2007). Latin American Cyberculture and Cyberliterature. Liverpool: Liverpool University Press.

Touraine, Jean-Louis (1985). Hors de la bulle. París: Flammarion.

Trésor de la Langue Française Informatisé (2011) [http://atilf.atilf.fr].

Yehya, Naief (2010). "La belleza y el Cyborg. El ideal de la belleza, su representación y la manipulación de la carne para acariciarlo", Disciplinas y prácticas corporales. Una mirada a las sociedades contemporáneas, edición de Elsa Muñiz, Barcelona-México: Anthropos-UAM-Azcapotzalco, 147-164.

Young, Robert (1995). Colonial Desire: Hybridity in Theory, Culture and Race. Londres: Routledge. 